\title{
Is TCI sufentanil the optimal choice of opioid during general anesthesia for endoscopic resection of pituitary adenomas?
}

\author{
Tomasz Napiórkowski, Jacek Kunicki, Agata Andruszak - Kot, Jacek Puściński, Małgorzata Symonides \\ Department of Anaesthesiology and Intensive Care \\ The Maria Skłodowska - Curie Memorial Cancer Center, Warsaw, Poland
}

\begin{abstract}
Background
During endoscopic resections of pituitary adenomas a steady and blood-free surgical field is required. This is ensured, among other factors, by maintaining systolic and mean blood pressure at a pre-specified and stable level and thus preventing large fluctuations in cerebral blood flow. After surgery the patient must almost immediately regain full awareness in order to allow for early neurological assessment.
\end{abstract}

The aim of the study was to examine whether use during general anesthesia sufentanil administered by TCI (traget controlled infusion) as an analgetic component is an optimal anesthetic procedure in the case of endoscopic resection of the pituitary adenoma.

The optimal procedure was determined that ensures hemodynamic stability during the resection stage, i.e. systolic blood pressure at 100 $110 \mathrm{mmHg}$ and perfusion mean arterial pressure.

\begin{abstract}
Materials and Methods
The study group included 45 patients aged from 26 to 84 years - 25 women and 20 men who were to undergo endoscopic resection of pituitary adenoma under general anesthesia. 23 of the patients had diagnosed hypertension, which accounted for $51 \%$ of cases.
\end{abstract}

The induction of general anesthesia was performed using propofol, and exceptionally etomitade in two cases and thiopental in three cases. To maintain the general anesthesia, an inhaled anesthetic - sevoflurane was used, and for the relaxation of skeletal muscle, rocuronium was used.

The opioid used was sufentanil, which was administered by an infusion pump using the TCI (target controlled infusion) method. This method uses the Gepts pharmacokinetic model contained in the TCI pump software, which adjusts the amount of administered drug depending on the target concentration of the drug in the effector set by the anesthesiologist in order to achieve the desired clinical effect.

For sufentanil, these are values in the range of 0.2 to $2 \mathrm{ng} / \mathrm{ml}$ of effector tissue.

The concentration of sufentanil during the induction and intubation of the patient was in each case $0.4 \mathrm{ng} / \mathrm{ml}$ of effector tissue.

Then the target concentration of sufentanil was adjusted in such a way that during the operation the systolic blood pressure was maintained at the level of approximately $100-110 \mathrm{mmHg}$ and the average blood pressure at the perfusion level.

\section{Results}

In 41 cases haemodynamic stability was maintained - systolic arterial pressure (SBP) in the resection stage in individual patients ranged from 85 to $110 \mathrm{mmHg}$, whereas mean arterial pressure (MAP) from 67 to $80 \mathrm{mmHg}$.

In two cases, due to the low values of SBP and MAP, ephedrine and noradrenaline were administered, respectively.

Hypertension requiring the administration of urapidil occurred in two cases.

Two patients had to be administered naloxone in order to rapidly reverse the opioid effect during emergence the patient.

Mean duration of anesthesia was 111 minutes (range $57.5-275 \mathrm{~min}$ ) and the average total dose of sufentanil was $54.1 \mu \mathrm{g}$ (range 27.9 - 116 $\mu \mathrm{g})$.

On average, the maximum applied concentration of sufentanil in the effector was $0.45 \mathrm{ng} / \mathrm{ml}$ ( $\mathrm{range} 0.4-0.7 \mathrm{ng} / \mathrm{ml}$ ).

The mean time from the end of surgery to extubation of a fully conscious patient was 12 min (range $2.5-30 \mathrm{~min}$ ).

\section{Conclusions}

TCI sufentanil appears to be an optimal opioid for general anesthesia during endoscopic surgery of pituitary adenomas.

Sufentanil provides analgesia with stable systolic pressure, which ensures a blood-less surgical field operative and maintains stable brainperfusion blood pressure.

The pharmacokinetic profile of sufentanil allows for rapid neurological recovery and evaluation of the patient regardless of the duration of anesthesia. The post-operative analgesic effect of sufentanil prevents the need for opioid administration during the postoperative period.

It was observed that at a sufentanil level of about $0.15 \mathrm{ng} / \mathrm{ml}$ patient is fully aware, has its own respiratory drive and does not complain of pain.

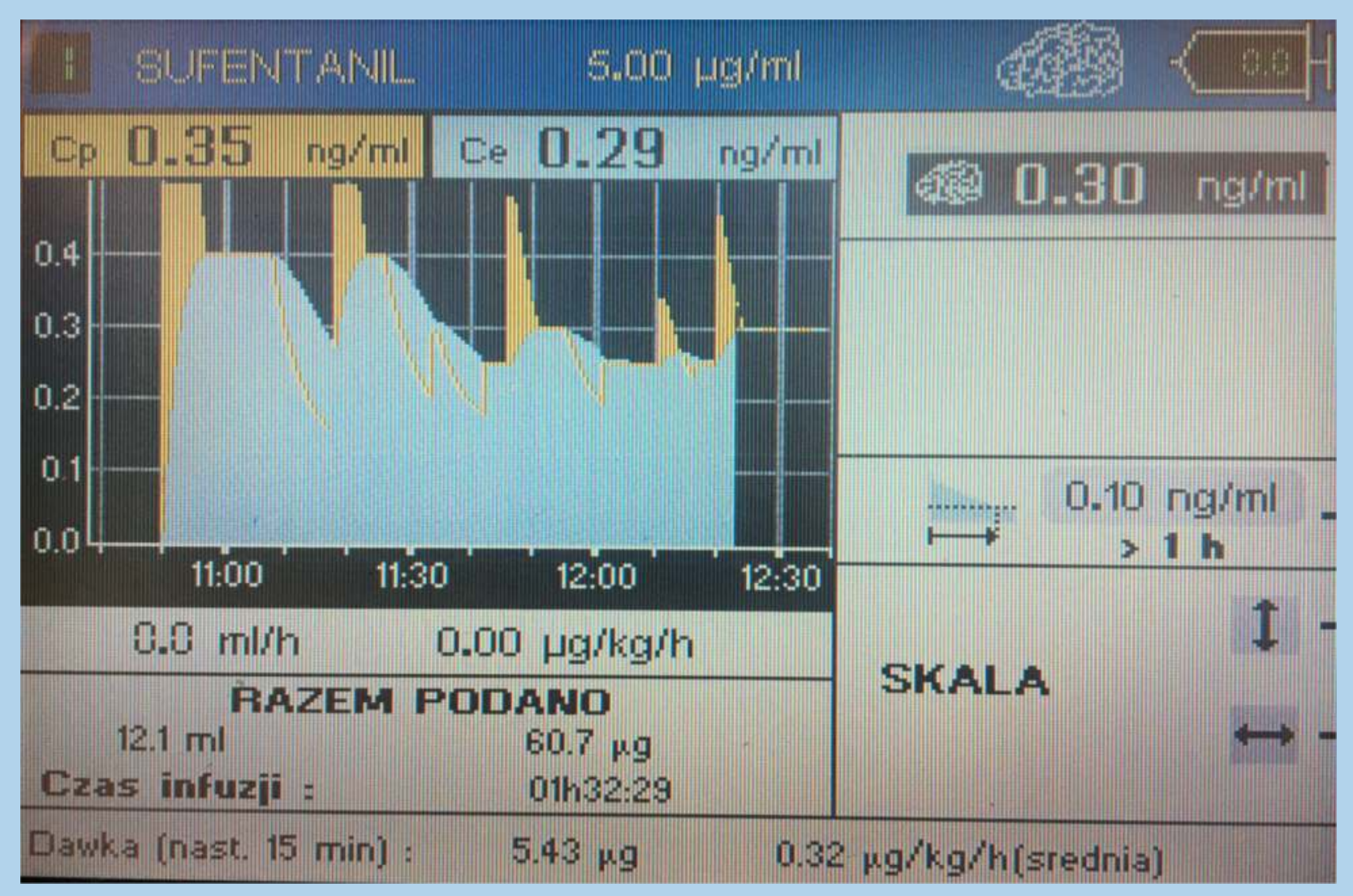

\section{References}

1. Cafiero, Tullio \& Mastronardi, P. (2000). [Sufentanyl in balanced anesthesia for neurosurgery. Comparative study with fentanyl]. Minerva anestesiologica. 66. $787-91$.

2. P Martorano, P \& Aloj, F \& Baietta, S \& Fiorelli, A \& Munari, M \& Paccagnella, F \& Rosa, Giovanni \& Scafuro, Mariantonietta \& Zei, E \& Falzetti, G \& Pelaia, Paolo. (2008). Sufentanil - propofol vs remifentanil - propofol during total intravenous anesthesia for neurosurgery. A multicentre study. Minerva anestesiologica. 74. 233 - 43. 